\title{
Clinical Outcome of Patients With Malignant Tumors Associated With Mature Cystic Teratomas of the Ovary: A Retrospective Multicenter Italian Study
}

\author{
ANGIOLO GADDUCCI ${ }^{1}$, DANIELA GIULIANI ${ }^{2}$, STEFANIA COSIO $^{1}$, \\ ANDREA LISSONI $^{2}$, ANNA MARIA FERRERO ${ }^{3}$ and FABIO LANDONI ${ }^{2}$ \\ ${ }^{1}$ Department of Clinical and Experimental Medicine, \\ Division of Gynecology and Obstetrics, University of Pisa, Pisa, Italy; \\ ${ }^{2}$ Clinic of Obstetrics and Gynecology, San Gerardo Hospital, Monza, \\ Department of Medicine and Surgery, University of Milan Bicocca, Milan, Italy; \\ ${ }^{3}$ Department of Gynecology and Obstetrics, Mauriziano Hospital, University of Turin, Turin, Italy
}

\begin{abstract}
Background/Aim: The aim of the study was to assess the clinical outcome of patients with malignant transformation of an ovarian mature teratoma. Patients and Methods: This study was conducted on 23 patients who underwent primary surgery at three Italian Gynecological Centers. Histologically, nine (39.1\%) patients had squamous cell carcinoma, five $(21.7 \%)$ had a thyroid carcinoma, six (26.1\%) had a carcinoid, one (4.3\%) patient had papillary renal carcinoma, one (4.3\%) had medulloblastoma and one (4.3\%) had intestinal-type mucinous adenocarcinoma. Results: All six patients with stage I squamous cell carcinoma had no evidence of disease (NED) after a median time of 141 months. Of the three patients with stage IIb-IIIC squamous cell carcinoma, two had NED after 119 and 154 months, and one died of the disease 9 months after diagnosis. All five women with stage I thyroid carcinoma had NED after a median of 60 months. Of the six patients with stage I carcinoid, five had NED after a median of 168 months, whereas one died due to carcinoid heart disease. The three patients with stage I renal carcinoma, medulloblastoma and mucinous adenocarcinoma had NED after 24, 141 and 149 months, respectively. Conclusion: The
\end{abstract}

Present address: Department of Clinical and Experimental Medicine, Division of Gynecology and Obstetrics, University of Pisa, Pisa, Italy.

Correspondence to: Angiolo Gadducci, Department of Experimental and Clinical Medicine, Division of Gynecology and Obstetrics, University of Pisa, Via Roma 56, Pisa, 56127, Italy. Tel: +39 50992609, Fax: +39 50553410, e-mail: a.gadducci@ med.unipi.it

Key Words: Mature cystic teratoma, squamous cell carcinoma, thyroid carcinoma, carcinoid, surgery. clinical outcome of early-stage malignancies associated with mature ovarian teratomas is excellent following treatment.

Mature cystic teratomas of the ovary have an incidence of approximately $1.2-14.2$ cases per 100.000 women per year, accounting for $60 \%$ of all benign ovarian tumors, and may occur at any age, with the highest peak between 30 and 40 years $(1,2)$. These ovarian germ cell tumors undergo malignant transformation in approximately $2 \%$ of the cases, and squamous cell carcinoma is the most commonly associated cancer $(1,3,4)$. Less frequently encountered neoplasias are represented by mucinous carcinomas $(5,6)$, thyroid carcinomas $(3,5-9)$, carcinoids $(5,10)$, neuroectodermal tumors $(11,12)$ and sarcomas (13).

The preoperative detection of malignant transformation of an ovarian mature cystic teratoma is very difficult. The diagnostic accuracy of ultrasound, magnetic resonance imaging and computed tomography is still debated, and the diagnosis usually emerges only at the time of histological examination of surgical specimens (14-16).

The mainstay of therapy of squamous cell carcinoma arising in a mature cystic teratoma of the ovary consists of bilateral salpingo-oophorectomy, total hysterectomy and comprehensive surgical staging in early carcinoma and optimal debulking surgery in advanced carcinoma $(2,3,4)$. The role of postoperative platinum-based chemotherapy is uncertain in patients with stage I disease, whereas it is a standard of care in those with more advanced disease (1-4, $17,18)$. The treatment of thyroid carcinoma associated with a mature cystic teratoma of the ovary is even more debated and ranges from demolitive surgery with surgical staging to unilateral salpingo-oophorectomy or ovarian cystectomy, especially in women with disease apparently confined to the ovary $(7,19,20)$. Carcinoids are usually treated with surgery 
alone, ranging from cystectomy (10) to unilateral or bilateral salpingo-oophorectomy $(15,21)$ to hysterectomy with bilateral salpingo-oophorectomy with or without surgical staging (22-26). The present multicenter retrospective investigation assessed the clinical findings, treatment modalities and prognosis of patients with malignancies associated with an ovarian mature cystic teratoma.

\section{Materials and Methods}

The present study reviewed the records of 23 patients with malignant transformation within a mature cystic teratoma of the ovary who underwent surgery at the Departments of Gynecology and Obstetrics of the University of Pisa (between 2010 and 2017), of the University of Milano-Monza (between 1993 and 2017) and of the University of Torino (Mauriziano Hospital) (between 2000 and 2017). Some patients had been reported in previous publications $(3,25)$.

The hospital records, including surgical notes, pathological reports, and follow-up data, were collected using a common form with standardized items and a common database.

The tumor stage of each case was determined according to the International Federation of Gynecology and Obstetrics [FIGO] criteria. The baseline characteristics (patient age, tumor stage, histological type, type of surgery, post-surgical management) were reported for each case.

The evaluation of the clinical course of disease was based on clinical examination, chest x-ray, abdominal-pelvic ultrasound, and computed tomography scan. Additional investigations were performed when appropriate. All patients were periodically followed-up at regular scheduled intervals until December 2018.

\section{Results}

The median age of patients was 44 years (range $=21-78$ years). The median value of the largest diameter of the ovarian mass was $8.5 \mathrm{~cm}$ (range=3.5-35.0 cm). The histological types of tumors were as follows: nine $(39.1 \%)$ cases were squamous cell carcinomas, five $(21.7 \%)$ were thyroid carcinomas (3 papillary, 2 follicular), six (26.1\%) were carcinoids (1 insular, 2 strumal, 3 not specified), one ( $4.3 \%$ ) case was a type 2 papillary renal carcinoma, one case $(4.3 \%)$ was a medulloblastoma, and one $(4.3 \%)$ case was a well-differentiated intestinal - type mucinous adenocarcinoma. Type of surgery and tumor stage are shown in Table I. Surgery was performed through laparoscopy in 12 and laparotomy in 11 patients.

Peritoneal staging (omentectomy and peritoneal biopsies) or peritoneal debulking (omentectomy+peritoneal resections \pm bowel resection) and pelvic and/or aortic lymph node resection were performed in 9 and 6 patients, respectively, of the 9 patients with squamous cell carcinoma. Tumor stage was Ia in 5 patients $(55.6 \%)$, and one $(11.1 \%$ ) for each Ic, IIb, IIIb and IIIc stage. Of the 6 patients with stage I disease, 5 had no further treatment and 1 patient underwent adjuvant chemotherapy with paclitaxel+ carboplatin. All these patients were alive with no evidence of disease after a median time of
141 months (range, 14 to 192 months) from diagnosis. The patient with stage IIb disease and the patient with stage IIIc disease received combination chemotherapy with cisplatin+ etoposide+bleomycin and were alive with no evidence of disease after 119 and 154 months, respectively. The patient with stage IIIb disease was treated with conservative surgery and combination chemotherapy with ifosfamide+paclitaxel+ cisplatin, but she developed pelvic and abdominal recurrence 4 months after diagnosis. Then the patient underwent total hysterectomy and debulking surgery followed by chemotherapy with cisplatin+gemcitabine, but the tumor rapidly progressed and the patient died 5 months later.

Regarding the 5 patients with thyroid carcinoma, peritoneal staging and retroperitoneal staging were performed in 3 and 2 patients, respectively. The patient treated with unilateral salpingo-oohorectomy had previously undergone thyroidectomy followed by iodine-131 ablation for a papillary carcinoma of the thyroid gland (9). The patient treated with total hysterectomy and bilateral salpingooophorectomy, underwent thyroidectomy for a benign struma 5 months later. All the 5 women with thyroid carcinoma within a mature cystic teratoma had stage I disease, did not receive additional treatment, and were alive with no evidence of disease after a median time of 60 months (range $=38-203$ months).

All 6 patients with carcinoid had stage I disease and none of them received further therapy. Five patients, who had not carcinoid syndrome, were alive with no evidence of disease after median time of 168 months (range=154-230 months). The patient with insular carcinoid was found to have a severe tricuspidal and mitral valve damage at echocardiography performed 3 weeks after surgery for severe weariness and weakness (51). She died due to sepsis and complications of the superimposed mitral endocarditis.

The patient with type- 2 papillary renal carcinoma (9), the patient with medulloblastoma and the patient with intestinaltype mucinous adenocarcinoma, had stage I disease, received no additional therapy after surgery, and were alive with no evidence of disease after 24, 141 and 149 months, respectively.

\section{Discussion}

In agreement with the literature (1, 3), squamous cell carcinoma is the most common malignancy arising from a mature cystic teratoma of the ovary, accounting for $39.1 \%$ of the cases of the present series. Tumor stage is the strongest prognostic variable $(1,2,4,17,18)$. Tseng et al. (18) reported that 2-year disease-free survival was $100 \%, 100 \%, 30 \%$ and $0 \%$ for the patients with stage I, stage II, stage III and stage IV disease, respectively ( $p=0.0001)$. Similarly, Cheng et al. (17) found 5-year overall survival rates of $75.7 \%, 33.8 \%$, $20.6 \%$ and $0 \%$ for patients with stage I, stage II, stage III and stage IV disease, respectively, $(p<0.0001)$. In the present 
Table I. Type of surgery and FIGO stage of patients with malignant transformation arising within a mature cystic teratoma of the ovary.

\begin{tabular}{|c|c|c|}
\hline Histological type & Pts (n.) & FIGO stage \\
\hline \multicolumn{3}{|l|}{ Squamous cell carcinoma } \\
\hline Surgery & 9 & \\
\hline $\mathrm{TH}+\mathrm{BSO}+$ peritoneal staging* & 2 & Ia (n.2) \\
\hline $\mathrm{TH}+\mathrm{BSO}+$ peritoneal staging $+\mathrm{RP}$ staging $* *$ & 3 & Ia (n.2) Ic (n.1) \\
\hline $\mathrm{TH}+\mathrm{BSO}+$ peritoneal staging/RP debulking & 2 & $\operatorname{IIb}(\mathrm{n} .1)$ IIIc (n.1) \\
\hline USO + peritoneal staging + RP staging & 1 & Ia \\
\hline USO + peritoneal staging & 1 & IIIb \\
\hline $\begin{array}{l}\text { Thyroid carcinoma } \\
\text { Surgery }\end{array}$ & 5 & \\
\hline USO + peritoneal staging & 1 & Ia \\
\hline Cystectomy + peritoneal staging & 1 & Ia \\
\hline USO + peritoneal staging* + RP staging & 2 & Ia $(\mathrm{n} .2)$ \\
\hline $\mathrm{TH}+\mathrm{BSO}$ & 1 & Ia \\
\hline $\begin{array}{l}\text { Carcinoid } \\
\text { Surgery }\end{array}$ & 6 & \\
\hline Cystectomy & 1 & Ia \\
\hline USO & 1 & Ia (n.1) \\
\hline BSO & 2 & Ia $(\mathrm{n} .2)$ \\
\hline $\mathrm{TH}+\mathrm{BSO}+$ peritoneal staging $+\mathrm{RP}$ staging & 2 & Ia $(\mathrm{n} .2)$ \\
\hline $\begin{array}{l}\text { Type- } 2 \text { papillary renal carcinoma } \\
\text { Surgery }\end{array}$ & 1 & \\
\hline USO & 1 & Ia \\
\hline $\begin{array}{l}\text { Mucinous intestinal adenocarcinoma } \\
\text { Surgery }\end{array}$ & 1 & \\
\hline $\mathrm{TH}+\mathrm{BSO}+$ peritoneal staging $+\mathrm{RP}$ lymphadenectomy & 1 & Ic \\
\hline $\begin{array}{l}\text { Medulloblastoma } \\
\text { Surgery }\end{array}$ & 1 & \\
\hline USO $* * *$ & 1 & Ia \\
\hline
\end{tabular}

Peritoneal staging*: Omentectomy, peritoneal biopsies and peritoneal washing with or without appendectomy. RP staging**: pelvic \pm aortic lymph node dissection. ***USO during cesarean section; peritoneal staging and endometrial biopsy 1 month later. TH: Total hysterectomy; BSO: bilateral salpingo-oophorectomy; RP: retroperitoneal; USO: unilateral salpingo-oophorectomy.

investigation, all 6 patients with stage I disease, of which only one received adjuvant chemotherapy, were alive with no evidence of disease after a median follow-up time of longer than 11 years. One of the 3 patients with stage IIb-IIIc carcinoma died early of disease. In our opinion, patients with tumor confined to the ovary should undergo bilateral salpingooophorectomy, total hysterectomy and comprehensive surgical staging, whereas unilateral salpingo-oophorectomy should be reserved to highly selected young women. Few data are currently available in the literature about this conservative approach $(4,17,18)$. Optimal cytoreductive surgery followed by platinum-based combination chemotherapy is the standard treatment for advanced tumors $(2,4,17,18,27)$. Conversely, the role of adjuvant chemotherapy in patients with early disease is uncertain, and these women should be carefully followed-up $(1,4,16-18,27,28)$.

The patients with thyroid carcinoma, carcinoid, papillary renal carcinoma, medulloblastoma and intestinal-type mucinous adenocarcinoma included in the present investigation underwent various surgical approaches, ranging from cystectomy to demolitive surgery, and received no adjuvant therapy. All of them had stage I disease.

Thyroid-type carcinoma arises within mature cystic teratomas in approximately $0.1 \%$ of cases (7). There is no consensus on the surgical and postoperative treatment of patients with this malignancy $(7,19,20)$. In addition to gynecological surgery, some authors also recommend total thyroidectomy and iodine-131 ablation, when preservation of fertility is not required, in order to enable follow-up with serum thyroglobulin assay and iodine scan (7). This treatment modality is warranted in metastatic disease (29). The 5 patients of the present series had an excellent clinical outcome.

Primary carcinoids of the ovary account for $0.3-1.7 \%$ of all carcinoids and less than $0.1 \%$ of all ovarian cancers $(10,21$, $24,30)$. One-third of patients with insular carcinoid, which is the most common type, have carcinoid syndrome, whereas those with other histological types usually have not $(10,22)$. The treatment of carcinoids confined to the ovary consists of surgery alone, with 10-year overall survival rates of approximately $100 \%(10,23)$. These tumors can occasionally 
recur after a long interval, and therefore the patients, and especially those with carcinoid syndrome at diagnosis, should be carefully followed-up for an extended period of time (25). Five of the 6 patients of our series, who did not have carcinoid syndrome, were alive with no evidence of disease after a median follow-up of 14 years, whereas the remaining patient died of carcinoid heart disease. Serotonin-like substances released directly by the tumor into the systemic bloodstream may cause cardiac lesions in the absence of hepatic metastasis (25). Pellikka et al. (31), who performed echocardiography in 132 patients with carcinoid syndrome, found that tricuspidal regurgitation/stenosis was the more common heart disease followed by mitral regurgitation/stenosis. Patients with carcinoid heart disease had significantly higher mean pretreatment urinary 5-hydroxyindoleacetic acid levels and significantly worse survival when compared with those without cardiac involvement.

The prognosis of patients with advanced ovarian carcinoid, which usually undergo aggressive cytoreductive surgery, is poor, with a 5-year overall survival rate of approximately $33 \%(10,21)$. It is noteworthy that several pharmacological tools, including somatostatin analogs, chemotherapy, interferons, mammalian target of rapamycin inhibitors and radiolabeled somatostatin, have recently been investigated in this clinical setting (32).

Whereas neural tissue is found in approximately $80 \%$ of mature cystic teratomas, malignant transformation of neuroectodermal tissue is an exceptional event with few cases reported in the literature $(11,12)$. All these experienced an excellent prognosis after surgical resection alone. Similarly, our patient with medulloblastoma was alive with no evidence of disease after 141 months.

There are case reports of carcinomatosis after laparoscopic removal of a presumed benign mature cystic teratoma with evidence of malignant transformation at the pathological examination of surgical specimens (33-35). Therefore, if the patient is operated by laparoscopy, an endoscopic retrieval bag must be used to avoid the spillage of cyst content.

In conclusion, the results of the present retrospective investigation and the review of the literature data suggest that the clinical outcome, following surgery and/or chemotherapy, of patients with early-stage malignancy arising from a mature cystic teratoma of the ovary is excellent, whereas the prognosis of those with advanced disease is unsatisfactory. Given the rarity of these malignancies and lack of level I evidence on treatment modalities, a large multicenter database is strongly warranted to better establish surgical and postsurgical management $(2,32)$.

\section{Conflicts of Interest}

The Authors declare that they have no conflicts of interest regarding this study.

\section{Authors' Contributions}

Study concept: A.G.; Study design: A.G.; A.L.; A.M. F.; F. L.; Data analysis and interpretation: all Authors; Manuscript preparation: A.G.; Manuscript editing: A.G.; A.L.; A.M. F.; F. L.; Manuscript review: all Authors.

\section{References}

1 Rim SY, Kim SM and Choi HS: Malignant transformation of ovarian mature cystic teratoma. Int J Gynecol Cancer 16: 140144, 2006. PMID: 16445624. DOI: 10.1111/j.1525-1438. 2006.00285.x

2 Hackethal A, Brueggmann D, Bohlmann MK, Franke FE, Tinneberg HR and Münstedt K: Squamous-cell carcinoma in mature cystic teratoma of the ovary: systematic review and analysis of published data. Lancet Oncol 9: 1173-1180, 2008. PMID: 19038764. DOI: 10.1016/S1470-2045(08)70306-1

3 Gadducci A, Pistolesi S, Guerrieri ME, Cosio S, Carbone FG and Naccarato AG: Malignant transformation in mature cystic teratomas of the ovary: case reports and review of the literature. Anticancer Res 38: 3669-3675, 2018. PMID: 29848726. DOI: 10.21873/anticanres.12644

4 Gadducci A, Guerrieri ME and Cosio S: Squamous cell carcinoma arising from mature cystic teratoma of the ovary: a challenging question for gynecologic oncologists. Crit Rev Oncol Hematol 133: 92-98, 2019. PMID: 30661663. DOI: 10.1016/j.critrevonc.2018.10.005

5 Desouki MM, Fadare O, Chamberlain BK, Shakir N and Kanbour-Shakir A: Malignancy associated with ovarian teratomas: frequency, histotypes, and diagnostic accuracy of intraoperative consultation. Ann Diagn Pathol 19: 103-106, 2015. PMID: 25773307. DOI: 10.1016/j.anndiagpath. 2015.02.004

6 Black JD, Roque DM, Pasternak MC, Buza N, Rutherford TJ, Schwartz PE, McCarthy S and Ratner E: A series of malignant ovarian cancers arising from within a mature cystic teratoma: a single institution experience. Int J Gynecol Cancer 25: 792-797, 2015. PMID: 25790042. DOI: 10.1097/IGC.0000000000000431

7 DeSimone CP, Lele SM and Modesitt SC: Malignant struma ovarii: a case report and analysis of cases reported in the literature with focus on survival and I131 therapy. Gynecol Oncol 89: 543-548, 2003. PMID: 12798728.

8 Roth LM, Miller AW 3rd and Talerman A: Typical thyroid-type carcinoma arising in struma ovarii: a report of 4 cases and review of the literature. Int J Gynecol Pathol 27: 496-506, 2008. PMID: 18753973. DOI: 10.1097/PGP.0b013e31816a74c6

9 Middelbeek RJW, O’Neill BT, Nishino M and Pallotta JA: Concurrent intrathyroidal thyroid cancer and thyroid cancer in struma ovarii: a case report and literature review. J Endocr Soc 23: 396-400, 2017. PMID: 29264493. DOI: 10.1210/js.2017-00052

$10 \mathrm{Kim}$ JY: A carcinoid tumor arising from a mature cystic teratoma in a 25-year-old patient: a case study. World J Surg Oncol 14: 120, 2016. PMID: 27098182. DOI: 10.1186/s12957016-0867-8

11 Yoder N, Marks A, Hui P, Litkouhi B and Cron J: Low-grade astrocytoma wthin a mature cystic teratoma in an adolescent patient. J Pediatr Adolesc Gynecol 31: 325-327, 2018. PMID: 29107097. DOI: 10.1016/j.jpag.2017.10.005 
12 Serrano-Arévalo ML, Lino-Silva LS and Domínguez Malagón HR: Oligodendroglial cell proliferation arising in an ovarian mature cystic teratoma. Clinicopathological, immunohistochemical, and ultrastructural study of a case that may represent an oligodendroglioma. Ultrastruct Pathol 41: 62-66, 2017. PMID: 28029275. DOI: 10.1080/01913123. 2016.1261975

13 Kefeli M, Kandemir B, Akpolat I, Yildirim A and Kokcu A: Rhabdomyosarcoma arising in a mature cystic teratoma with contralateral serous carcinoma: Case report and review of the literature. Int J Gynecol Pathol 28: 372-375, 2009. PMID: 19483625. DOI: 10.1097/PGP.0b013e3181929269

14 Emoto M, Obama H, Horiuchi S, Miyakawa $\mathrm{T}$ and Kawarabayashi T: Transvaginal color Doppler ultrasonic characterization of benign and malignant ovarian cystic teratomas and comparison with serum squamous cell carcinoma antigen. Cancer 88: 2298-2304, 2000. PMID: 10820352.

15 Jung SE, Lee JM, Rha SE, Byun JY, Jung JI and Hahn ST: CT and MR imaging of ovarian tumors with emphasis on differential diagnosis. Radiographics 22: 1305-1325, 2002. PMID: 25540571. DOI: $10.4103 / 0976-7800.145169$

16 Patni R: Squamous cell carcinoma arising in mature cystic teratoma of ovary. J Midlife Health 5: 195-197, 2014. PMID: 25540571. DOI: 10.4103/0976-7800.145169

17 Chen RJ, Chen KY, Chang TC, Sheu BC, Chow SN and Huang SC: Prognosis and treatment of squamous cell carcinoma from a mature cystic teratoma of the ovary. Formos Med Assoc 107: 857-868, 2008. PMID: 18971155. DOI: 10.1016/S09296646(08)60202-8

18 Tseng CJ, Chou HH, Huang KG, Chang TC, Liang CC, Lai CH, Soong YK, Hsueh S and Pao CC: Squamous cell carcinoma arising in mature cystic teratoma of the ovary. Gynecol Oncol 63: 64-70, 1996. PMID: 8946873. DOI: 10.1006/gyno.1996. 0337

19 Goffredo P, Sawka AM, Pura J, Adam MA, Roman SA and Sosa JA: Malignant struma ovarii: a population-level analysis of a large series of 68 patients. Thyroid 25: 211-215, 2015. PMID: 25375817. DOI: $10.1089 /$ thy.2014.0328

20 Zhu Y, Wang C, Zhang GN, Shi Y, Xu SQ, Jia SJ and He R: Papillary thyroid cancer located in malignant struma ovarii with omentum metastasis: a case report and review of the literature. World J Surg Oncol 14: 17, 2016. PMID: 26791568. DOI: 10.1186/s12957-016-0776-x

21 Kopf B, Rosti G, Lanzanova G and Marangolo M: Locally advanced ovarian carcinoid. J Exp Clin Cancer Res 24: 313-316, 2005. PMID: 16110766.

22 De la Torre J, García A, Castellví J, López M and Gil A: Primary ovarian trabecular carcinoid tumour: a case report with an immunohistochemical study and a review of the literature. Arch Gynecol Obstet 270: 274-277, 2004. PMID: 12774237. DOI: 10.1007/s00404-003-0509-6

23 Guney N, Sayilgan T, Derin D and Ozcan D: Primary carcinoid tumor arising in a mature cystic teratoma of the ovary: a case report. Eur J Gynaecol Oncol 30: 223-225, 2009. PMID: 19480263.

24 Bai X, Li N, Wang F, Li S and Yu Q: Primary ovarian trabecular carcinoid tumor: a case report and literature review. Arch Gynecol Obstet 282: 407-411, 2010. PMID: 20652280. DOI: $10.1007 /$ s00404-010-1600-4
25 Buda A, Giuliani D, Montano N, Perego P and Milani R: Primary insular carcinoid of the ovary with carcinoid heart disease: Unfavourable outcome of a case. Int J Surg Case Rep 3: 59-61, 2012. PMID: 22288046. DOI: 10.1016/j.ijscr.2011. 10.014

26 Amano Y, Mandai M, Baba T, Hamanishi J, Yoshioka Y, Matsumura N and Konishi I: Recurrence of a carcinoid tumor of the ovary 13 years after the primary surgery: A case report. Oncol Lett 6: 1241-1244, 2013. PMID: 24179502. DOI: 10.3892/ol.2013.1530

27 Chiang AJ, La V, Peng J, Yu KJ and Teng NN: Squamous cell carcinoma arising from mature cystic teratoma of the ovary. Int J Gynecol Cancer 21: 466-474, 2011. PMID: 21430455. DOI: 10.1097/IGC.0b013e31820d3e5b

28 Goudeli C, Varytimiadi A, Koufopoulos N, Syrios J and Terzakis E: An ovarian mature cystic teratoma evolving in squamous cell carcinoma: A case report and review of the literature. Gynecol Oncol Rep 19: 27-30, 2016. PMID: 28050596. DOI: 10.1016/ j.gore.2016.12.005

29 Zekri JM, Manifold IH and Wadsley JC: Metastatic struma ovarii: late presentation, unusal features and multiple radioactive iodine treatments. Clin Oncol 18: 768-772, 2006. PMID: 17168212.

30 Boyraz G, Selcuk I, Guner G, Usubutun A and Gunalp GS: A primary insular type carcinoid tumor arising in a mature cystic teratoma of the ovary: a case report. J Clin Case Rep 2: 16, 2012. PMID: 29888753. DOI: 10.4172/2165-7920.10001033

31 Pellikka PA, Tajik AJ, Khandheria BK, Seward JB, Callahan JA, Pitot HC and Kvols LK: Carcinoid heart disease. Clinical and echocardiographic spectrum in 74 patients. Circulation 87: 11881196, 1993. PMID: 7681733

32 Reed NS, Gomez-Garcia E, Gallardo-Rincon D, Barrette B, Baumann K, Friedlander M, Kichenadasse G, Kim JW, Lorusso D, Mirza MR and Ray-Coquard I: Gynecologic Cancer InterGroup (GCIG) consensus review for carcinoid tumors of the ovary. Int J Gynecol Cancer 24: S35-41, 2014. PMID: 25341578. DOI: $10.1097 /$ IGC .0000000000000265

33 Wang PH, Yen MS, Juang CM, Chen YJ, Chao HT and Yuan $\mathrm{CC}$ : Intraperitoneal cancer spread after laparoscopic cystectomy for mature teratoma with malignant transformation. Eur $\mathrm{J}$ Gynaecol Oncol 23: 131-132, 2002. PMID: 12013109.

34 Wen KC, Hu WM, Twu NF, Chen P and Wang PH: Poor prognosis of intraoperative rupture of mature cystic teratoma with malignant transformation. Taiwan J Obstet Gynecol 45: 253-256, 2006. PMID: 17175475. DOI: 10.1016/S10284559(09)60236-9

35 Sinha A and Ewies AA: Ovarian mature cystic teratoma: challenges of surgical management. Obstet Gynecol Int 2016: 2390178, 2016. PMID: 27110246. DOI: 10.1155/2016/2390178

Received March 20, 2019

Revised April 13, 2019 Accepted April 16, 2019 